\title{
A New Model of Heart Failure Post-Myocardial Infarction in the Rat
}

\author{
Honglei $\mathrm{Li}^{1,2}$, Jiansheng Huang ${ }^{3}$, Caihong Liu ${ }^{1}$, Zhentao Zhang ${ }^{3}$, Kuiquan Song ${ }^{2}$, Ke Ma ${ }^{1}$, Connor W. Dennewitz ${ }^{3}$, Shijun \\ Wang $^{1,4}$ \\ ${ }^{1}$ College of Traditional Chinese Medicine, Shandong University of Traditional Chinese Medicine ${ }^{2}$ Department of Intensive Care Unit, Jinan Municipal \\ Hospital of Traditional Chinese Medicine ${ }^{3}$ Department of Medicine, Vanderbilt University Medical Center ${ }^{4}$ Shandong Co-Innovation Center of Classic TCM \\ Formula, Shandong Provincial Education Department
}

\section{Corresponding Author}

Shijun Wang

pathology@163.com

\section{Citation}

Li, H., Huang, J., Liu, C., Zhang, Z., Song, K., Ma, K., Dennewitz, C.W., Wang, S. A New Model of Heart Failure Post-Myocardial Infarction in the Rat. J. Vis. Exp. (172), e62540, doi:10.3791/62540 (2021).

\section{Date Published}

June 10, 2021

DOI

$10.3791 / 62540$

URL

jove.com/video/62540

\section{Abstract}

Ligation of the left anterior descending (LAD) coronary artery has been widely used to establish the rat model of heart failure (HF) post myocardial infarction (MI). However, the disadvantages of this model include high mortality rate after ligation and larger variations both in the infarct size and the degree of impaired cardiac function. In addition, a ventilator or exteriorization of the heart is indispensable for the previous models, which complicates the procedure during the ligation. In this study, we developed a reliable and reproducible model without the ventilator or exteriorization of the heart by ligating the LAD coronary artery. Four weeks after the procedure, we found that the serum concentrations of CK-MB, NT-proBNP, and Renin, which were used to assist diagnoses of $\mathrm{Ml}$ and $\mathrm{HF}$, were significantly higher in the MI group compared to the sham group. In contrast, the value of left ventricle ejection fraction (LVEF) in the MI group was obviously less than in the sham group. Furthermore, the infarct size and cardiac fibrosis area were individually confirmed and quantitatively analyzed by TTC staining and Masson's trichrome staining. Smaller variations were found in either infarct size or fibrosis area in the MI group, which helped to develop a reliable and reproducible model of HF post-MI. This new model of HF post-MI in the rat is vital for studying the potential mechanisms of MI and HF. This new method can be used to develop the new drug for treatment of $\mathrm{MI}$ and $\mathrm{HF}$ in rats by using pharmacological strategies.

\section{Introduction}

Heart failure (HF) is considered a global public health issue that affects over 26 million patients and the prevalence is still increasing ${ }^{1}$. HF is defined as when the heart does not pump blood as well as it should. There are many risk factors for HF, including ischemic heart disease, diabetes, hypertension, LV hypertrophy, obesity, smoking, etc. $2,3,4$. 
Myocardial infarction (MI) is one of the most common causes of $\mathrm{HF}^{5}$. For almost 50 years, $\mathrm{HF}$ following $\mathrm{Ml}$ has been the major driver of late morbidity, mortality, and healthcare $\operatorname{cost}^{6}$. For better understanding of the pathophysiological mechanism of $\mathrm{HF}$ and to prevent and treat $\mathrm{HF}$ more effectively, large numbers of animal HF models post-MI have been developed for preclinical study. Rats are typically used due to easy maintenance, lower costs, ability for controls, and high homology with humans ${ }^{7,8,9}$. Left coronary artery (LCA) ligation, especially the left anterior descending (LAD) branch ligation, is widely used to develop the model of HF post-MI in the rat ${ }^{8}$.

However, surgeries that induce $\mathrm{MI}$ in rats are often technically challenging to perform due to high variation, complicated operation, and high mortality ${ }^{10,11}$. Even though quite a few procedures have been reported, the vast majority of surgeries required either ventilation after tracheal intubation or exteriorization of the heart. Those methods increased the cost of surgery, made the operation more difficult, and reduced the safety of the animal. In addition, the extent of HF is dependent on the site of the ligation of LCA. The anatomic diversity of the LCA in rats leads to inconsistency in infarction sizes and functional parameters in the rat model. Different ligation sites of LCA cause special infarct size, which determines the degree of $\mathrm{HF}$ and the survival rate.

In this study, we aimed to create a reliable and reproducible HF post-MI model. Our findings allowed for surgeries of rats without a ventilator or exteriorization of the heart, which resulted in a high survival rate. Also, the relatively fixed site of the ligation of LAD led to less variations of HF 4 weeks post-surgery. Furthermore, we have evaluated the effects of the HF model by using histopathological staining, biochemical detection, and cardiac function measurement, which helped to study the potential mechanisms behind the disease and develop new drugs to treat HF.

\section{Protocol}

The protocol was approved by the Animal Ethics Research Committee of Shandong University of Traditional Chinese Medicine.

NOTE: Adult male Sprague-Dawley rats, 10-weeks-old weighing 180-220 g, were randomly divided into 2 groups, sham $(n=25)$ and MI $(n=35)$. Animals were kept on a 12/12 $\mathrm{h}$ light/dark cycle and received water and food ad libitum.

\section{Anesthesia and continuous supply of oxygen}

1. Place rats into an induction chamber and anesthetize with $3 \%$ isoflurane.

1. To confirm whether the anesthetization was ready, observe the toe pinch reflex. The anesthetization is sufficient when the toe pinch reflex disappears.

2. Individually move the rats to a small temperaturecontrolled surgery board. Tape the rat's paws to the board.

3. Attach a mask to the rat face that provides a constant supply of $3 \%$ isoflurane and $1 \%$ oxygen.

\section{Induction of MI without ventilation or exteriorization of heart}

1. Use depilatory creams to remove the thorax hairs for sterilization.

2. Use a sterile drape with a $1 \mathrm{~cm} \times 3 \mathrm{~cm}$ hole at the surgical field of the rat.

3. Confirm sufficient depth of anesthesia with a toe pinch before operating. 
4. Open the chest with a transverse $2.5 \mathrm{~cm}$ incision between the third and fourth intercostal space on the left edge of the sternum without cutting the ribs.

5. Separate the pectoralis major and pectoralis minor muscles by using two vascular forceps without cutting the tissue or small blood vessels. Position the Weitlaner selfretaining retractor into the thorax to separate the third and fourth ribs to gain enough exposure of the heart while keeping rib integrity.

6. Delicately dissect the pericardium with two forceps.

7. Use a $6 / 0$ nylon suture with a curved needle to permanently ligate the LAD $2.0-2.5 \mathrm{~mm}$ below the midpoint of the conus arteriosus and left atrial appendage connection. $L A D$ can be easily recognized and found in some rats.

1. Confirm appropriate ligation of the LAD after the anterior wall of the left ventricle turned pale.

2. In the sham group, pass only the suture under the LAD, but do not ligate.

8. Remove the retractor and squeeze the air in the thorax out before suturing the muscle and skin layer by layer.

9. After surgery, place the rats on a $37^{\circ} \mathrm{C}$ heating pad for recovery. Monitor all animals, and provide ketoprofen (5 $\mathrm{mg} / \mathrm{kg} \mathrm{SC}$ ) for analgesia every 24 hours up to 72 hours. NOTE: For technical reasons, rats with smaller body weights (less than $250 \mathrm{~g}$ ) are preferred for this operation. The time of keeping chest open, from step 2.4-2.8, was limited to 5 minutes.

\section{Assessment of cardiac function}

NOTE: To confirm whether HF developed successfully, the cardiac function was measured 4 weeks post-procedure using a TE7 ultrasound system equipped with a $13 \mathrm{MHz}$ electronic transducer. During the echocardiography test, the rats were under the same anesthesia protocol used for the initial procedure.

1. After shaving the anterior chest hair, position the rats on the board used for the previous operation.

2. Place the probe on the anterior chest wall and obtain images from the left parasternal long-axis (PLAX) views of the left ventricle (LV) (at the level of papillary muscles).

3. Measure the left ventricular internal dimensions at enddiastole (LVIDd) and end-systole (LVIDs) by M-mode from three consecutive cardiac cycles.

4. Calculate the LV ejection fraction (LVEF) and the LV fractional shortening (LVFS) by the software of the machine.

\section{Further analysis of the HF model 4 weeks after LAD ligation}

1. Sacrafice the rats 4 weeks after LAD ligation by administering an overdose of isoflurane. Measure the serum concentrations of $\mathrm{CK}-\mathrm{MB}, \mathrm{N}$-terminal pro-brain natriuretic peptide (NT-proBNP), Renin, Angiotensin (Angll), Aldosterone (ALD), TNF- $\alpha$, VEGF, IL-6, and HIF-1 $\alpha$ by using the ELISA kits according to manufacturer's protocol.

2. To harvest the hearts for histopathological analysis, perfuse the rat hearts with $4{ }^{\circ} \mathrm{C}$ physiologic saline before being removed.

3. Set the hearts in $10 \%$ formalin for 24 hours, embed in paraffin, and slice at $5.0 \mu \mathrm{m}$ thick sections transversely along the left ventricular axis. 
4. Mount all sections of the heart on glass slides and individually stain with Hematoxylin and Eosin (HE) and Masson's trichrome.

5. For the purpose of infarct size measurement, stain hearts with 2,3,5-Triphenyl tetrazolium chloride (TTC).

1. Slice the hearts transversally at $3 \mathrm{~mm}$ thick from apex to base in a semi-frozen state and incubated in $2 \%$ TTC solution for 20 minutes at $37^{\circ} \mathrm{C}$.

2. When the color was established, fix slices in $4 \%$ paraformaldehyde solution for 15 minutes and take pictures.

3. Mark the infarct size and calculate by using ImageJ. Express the infarct size as a percentage of the infarct area versus the total LV area.

6. To quantitatively analyze the collagen content in the middle of the infarcted area of LV, stain the rat heart tissue sections with Masson's trichrome.

7. After staining, scan the images and analyze. Evaluate the degree of fibrosis by the collagen volume fraction (CVF) which was expressed as a percentage of the fibrotic area versus the total LV area.

\section{Representative Results}

The procedure was performed with a low mortality rate. The key instruments used for this experiment are the Weitlaner Self-Retaining Retractor $(13.5 \mathrm{~cm})$ and isoflurane vaporizer shown in Figure 1. The MI model was developed without ventilation or exteriorization of the heart as described in the protocol. During the whole procedure, all ribs were kept in integrity and the entire procedure took about 10 minutes. The schematic diagram of the surgical ligation site is shown in Figure 2. In this study, 2 rats died from ventricular fibrillation during the ligation procedure in the MI group, and 1 rat died due to bleeding after the heart was accidently pierced by the curved needle in the sham group. The mortality rate was about $5 \%$ throughout the experiment.

The cardiac function of rats was reduced significantly in the $\mathrm{Ml}$ group, and HF was successfully developed. Echocardiographic measurements were obtained in rats 4 weeks after the procedure to evaluate the effects of the HF models (Figure 3). Based on the 2016 ESC guidelines for the diagnosis and treatment of acute and chronic heart failure $^{12}$, the rats with LVEF less than $50 \%$ are considered as successful HF models. The main parameters related to heart failure were summarized in Table 1. When comparing the MI group to the sham group, LVEF in the MI group reduced significantly $(32.7 \% \pm 8.0$ VS $75.3 \% \pm 4.9, \mathrm{P}<0.001)$. These significant decreases of FS and increases of LVIDd and LVIDs in the MI group were good signs of HF. Additionally, the changes in the ventricular structure were seen from the ultrasound images (Figure 3). The chamber of LV got larger, and the wall of LV got thinner and stiffer in the MI group compared to the sham.

\section{Assessment of biomarkers of MI and HF post-MI by ELISA} As shown in Figure 4 and Figure 5, the serum concentrations of the cardiac marker CK-MB used to assist the diagnosis of MI rose significantly by more than 3 times in the MI group. Meanwhile, some parameters related to heart failure such as renin, Angll, and ALD serum concentrations were higher compared to the sham group 4 weeks post-procedure. The concentrations of NT-proBNP in the MI group was 13 times higher than in the sham group. Also, the concentrations of pro-inflammatory cytokines in the MI group, including TNF$\alpha$ and IL- 6 were increased by $400 \%$ and $300 \%$ compared to in the sham group. Meanwhile, as the representative angiogenesis-related factors like VEGF, and HIF-1a were 
also significantly higher by 2 and 5 times in the MI group compared to in the sham group.

\section{Morphological alterations and histopathology analyses}

In the Ml group, the morphological analysis of the hearts revealed a thin and pale LV wall as well as fibrous scar formation (Figure 6). Additionally, MI was also verified using TTC staining and the infarct size was tested (Figure 6). The infarct size was $40.7 \pm 4.4 \% 4$ weeks after the procedure in the MI group, which showed the reliability and stability of the new method of HF post-MI. For HE staining, microscopic evaluation displayed a neat arrangement of myocardial fibers without inflammatory change in the sham group. However, the myocardial fibers became a loose and irregular arrangement with inflammatory cellular infiltrates in the MI group (Figure 7a). In addition, Masson's trichrome staining revealed the areas of cardiac fibrosis were increased in the MI group (Figure 7a), and the collagen volume fraction (CVF) was $39.2 \pm 6.9 \%$ in the Ml group. The Masson's trichrome staining results were consistent with the TTC staining, which further confirmed the successful development of MI and HF models (Figure $7 b, 7 c$ ).

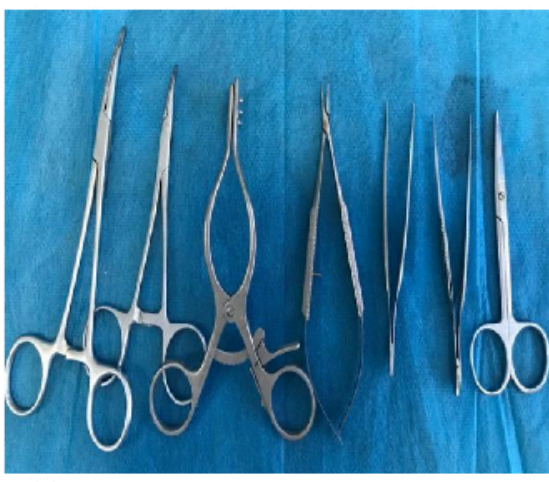

(a)

(b)

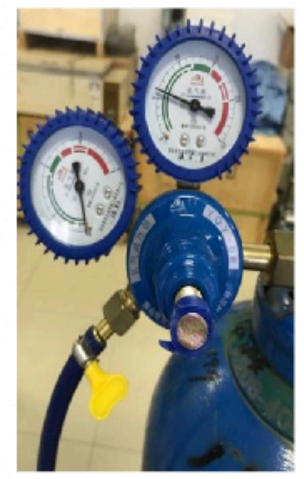

(c)

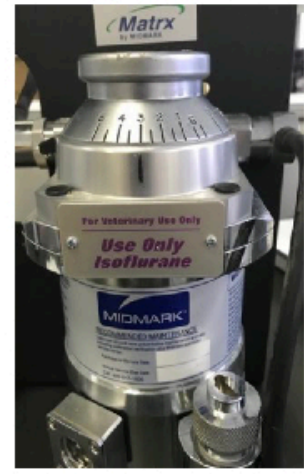

Figure 1. Key instruments were used to establish the MI model. (a) The Weitlaner Self-Retaining Retractor (13.5 cm) (Third from left); (b) The oxygen supply equipment; (c) The isoflurane vaporizer. Please click here to view a larger version of this figure. 


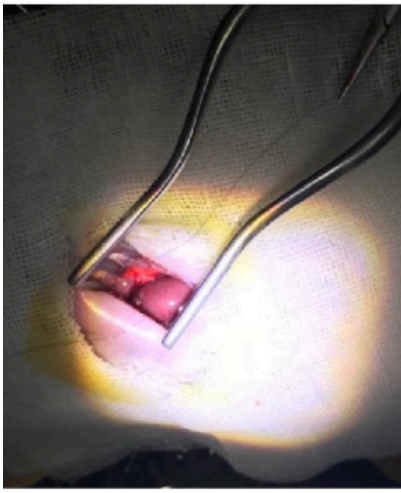

(a)

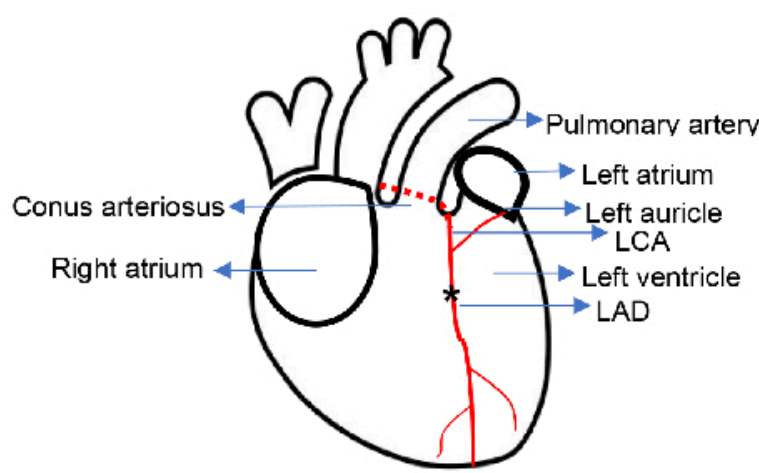

(b)

Figure 2. Experimental schematic. (a) Exposure of the heart with Weitlaner Self-Retaining Retractor; (b) The ligation location is indicated. Asterisk illustrates the ligation position. LCA, left coronary artery; LAD, left anterior descending. Please click here to view a larger version of this figure.

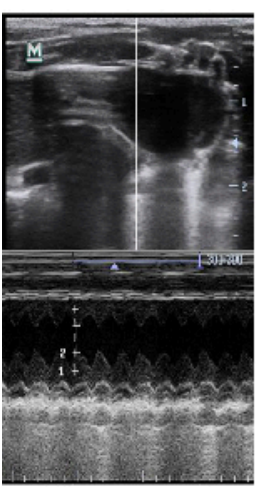

Sham

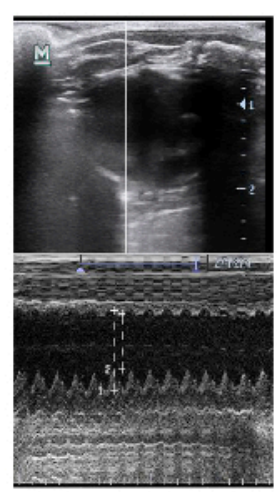

MI

(a)

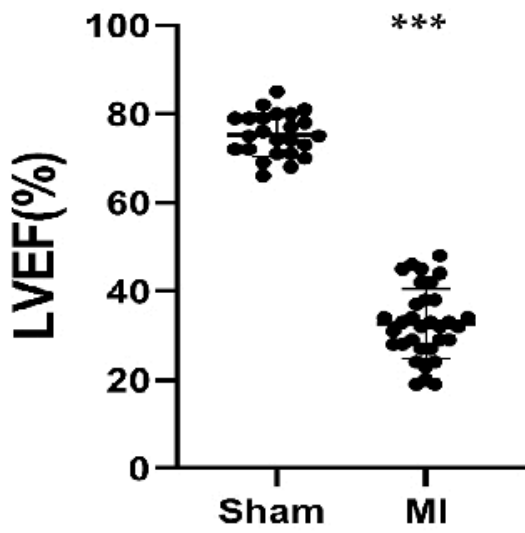

(b)

Figure 3. Echocardiographic measurements. (a) The representative images of left ventricle structures from the sham and MI group tested by M-Mode during 3 cardiac cycles after 4 weeks of the procedure; (b) LVEF of rats after 4 weeks of the procedure from the sham $(n=24)$ and MI $(n=33)$ group. MI, myocardial infarction. LVEF, left ventricle ejection fraction. ${ }^{* * *} P<$ 0.001 compared with the sham group. Please click here to view a larger version of this figure. 


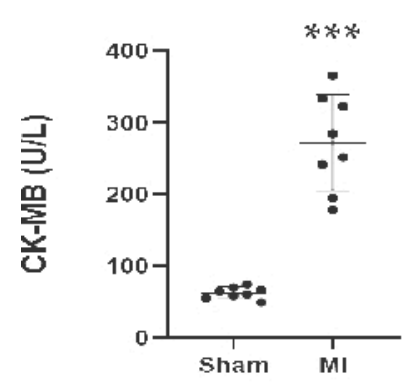

(a)

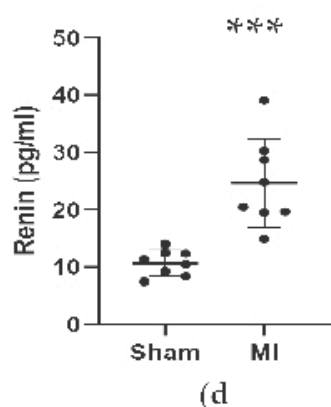

(d

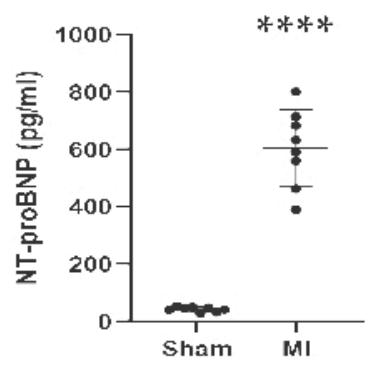

(b)

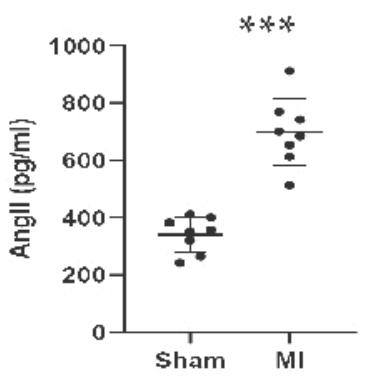

(e)

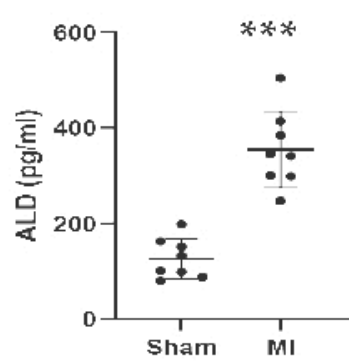

(c)

Figure 4. Concentrations of CK-MB, NT-proBNP, ALD, Renin and Angll were increased 4 weeks after LAD ligation.

Data were expressed as mean \pm SD ( $n=8$ animals in each group). MI, myocardial infarction; LAD, left anterior descending. ${ }^{* * *} \mathrm{P}<0.001$ compared with the sham group. ${ }^{* * * *} \mathrm{P}<0.0001$ compared with the sham group. Please click here to view a larger version of this figure. 


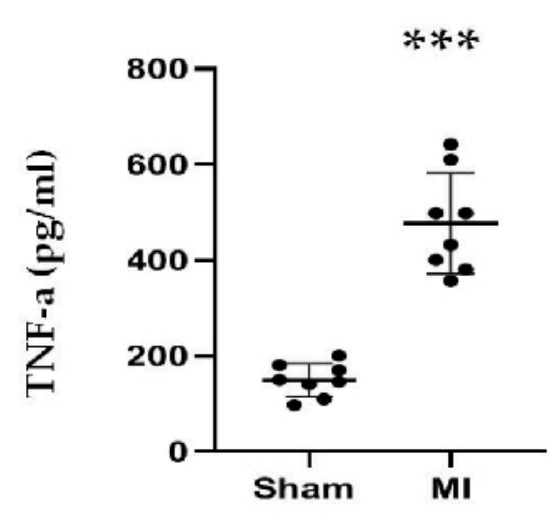

(a)

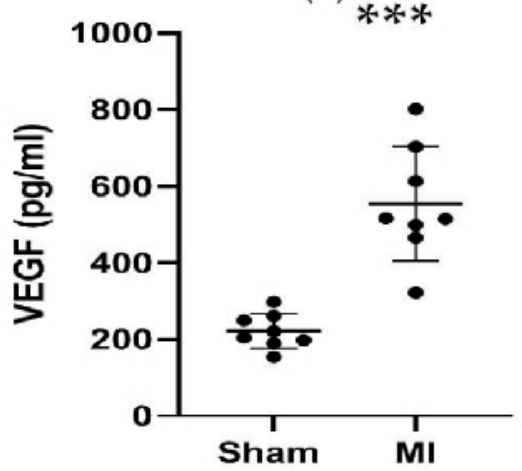

(c)

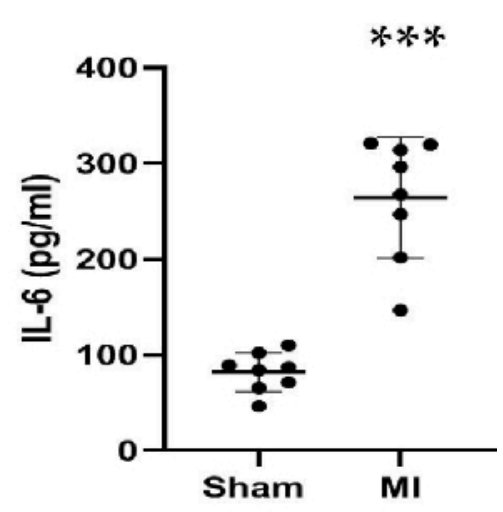

(b)

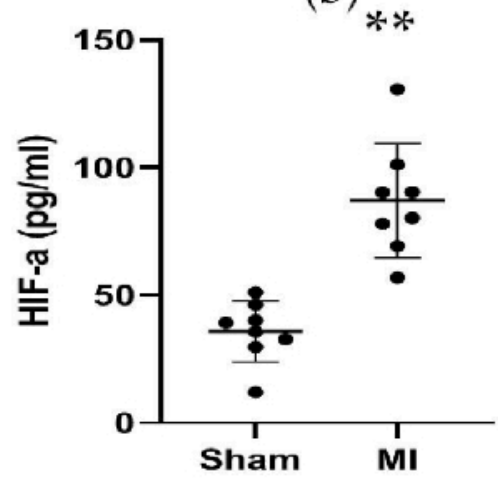

(d)

Figure 5. Concentrations of TNF- $\alpha$, IL-6,VEGF, and HIF- $\alpha$ were increased 4 weeks after LAD ligation. (a, b) Concentrations of TNF- $\alpha$ and IL- 6 associated with inflammation response were increased 4 weeks after LAD ligation; (c, d) Concentrations of VEGF and HIF- $\alpha$ associated with angiogenesis were increased 4 weeks after LAD ligation. Data are expressed as mean $\pm S D$ ( $n=8$ animals in each group). LAD, left anterior descending; MI, myocardial infarction. ${ }^{* *} P<0.01$ compared with the sham group. ${ }^{* * *} \mathrm{P}<0.001$ compared with the sham group. Please click here to view a larger version of this figure. 


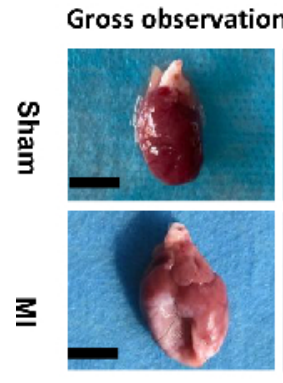

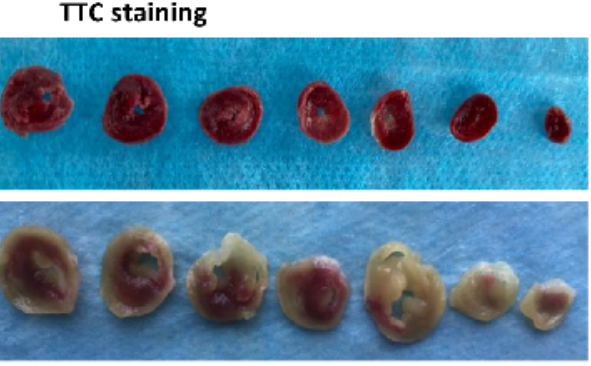

(a)

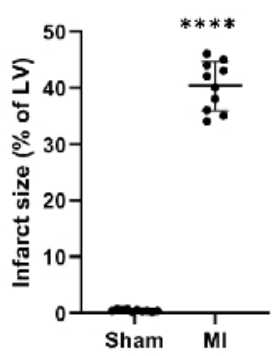

(b)

Figure 6. Morphological analysis of the hearts. (a) Gross observation and histology of rat hearts from sham and Ml group 4 weeks after procedure. The MI heart showed a thinner and bigger left ventricle wall compared to the sham; For the TTC staining hearts of the sham and Ml group, viable tissue was stained red and infarct area was pale and unstained. (b) MI infarct size was expressed as the percentage of the infarct area relative to the whole LV. Data are expressed as mean \pm SD ( $\mathrm{n}=10$ animals in each group).MI, myocardial infarction; TTC, triphenyl tetrazolium chloride; LV, left ventricle. Scale bar= $5 \mathrm{~mm} .{ }^{* * * *} \mathrm{P}<0.0001$ compared with the sham group. Please click here to view a larger version of this figure. 


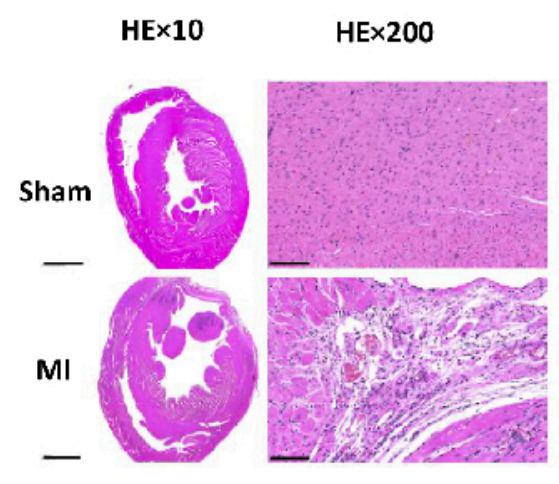

(a)

\section{Masson's trichrome}

staining $\times 10$

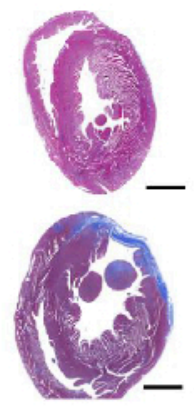

(b)

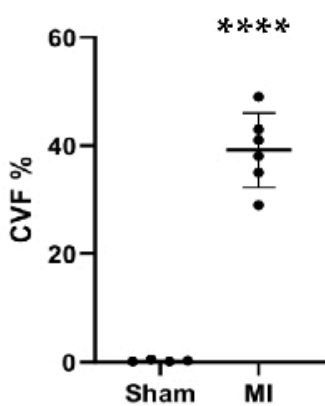

(c)

Figure 7. HE and Masson's trichrome staining of the rat heart tissue 4 weeks after the procedure. (a) The MI heart LV wall became thinner than the sham group (HE×10, Scale bar $=2 \mathrm{~mm}$ ). Microscopic evaluation displayed a neat arrangement of myocardial fibers without inflammatory change in the sham group and displayed a loose and irregular arrangement with inflammatory cellular infiltrates in the MI group (HE×200, Scale bar $=100 \mu \mathrm{m})$; (b) Masson's trichrome staining of heart tissue shows the myocardial fibrosis as blue in the MI group (Scale bar = $2 \mathrm{~mm}$ ). (c) Collagen volume fraction for Masson's trichrome staining in left ventricular tissue slices from the sham and MI groups. Data are expressed as mean \pm SD $(n=6$ animals in MI group). HE, hematoxylin and eosin; MI, myocardial infarction; LV, left ventricle. ${ }^{* * * *} \mathrm{P}<0.0001$ compared with the sham group. Please click here to view a larger version of this figure.

\begin{tabular}{|c|c|c|}
\hline Parameters & Sham group(n=24) & MI group(n=33) \\
\hline LVIDd (mm) & $8.3 \pm 1.3$ & $10.1 \pm 2.9^{*}$ \\
\hline LVIDs (mm) & $4.1 \pm 0.9$ & $7.7 \pm 1.5^{\star * *}$ \\
\hline FS (\%) & $42.5 \pm 7.8$ & $22.2 \pm 4.4^{* * *}$ \\
\hline
\end{tabular}

Table 1: Echocardiographic data of rats in the sham and MI group 4 weeks after LAD ligation. Data were expressed as mean \pm SD. MI, myocardial infarction; LVIDd, left ventricular internal dimensions at end-diastole at end-diastole; LVIDs, left ventricular internal dimensions at end-systole; FS\%, percent fractional shortening. ${ }^{*} \mathrm{P}<0.05$, compared with the sham group. ${ }^{* * *} \mathrm{P}<0.001$ compared with the sham group.

\section{Discussion}

Although there have been many existing models of $\mathrm{Ml}$ or HF in rodents ${ }^{13,14,15}$, this study has developed a novel and efficient LAD ligation procedure for inducing HF post-MI in rats. However, in this new rat HF model, the needs for intubation and ventilation or exteriorization of the heart were 
eliminated, which significantly increased the survival rate of the rats. To develop this new rat HF model, the induction of $\mathrm{Ml}$ is a crucial step. In comparison to the conventional protocols which usually involved intubation and ventilation or exteriorization of the heart during ligation $9,16,17$, we developed an improved approach with neither ventilation nor exteriorization of the heart for the first time allowing for a higher survival rate. It is worth noting that the improved procedure leads to less tissue damage. Therefore, rats have a much faster post-surgery recovery and higher survival rate. The following two points allowed us to achieve these results: First, the continuous inhalation of oxygen during the whole procedure allowed for sufficient oxygenation and ligation without ventilation. Second, the Weitlaner selfretaining retractor, a key surgical instrument, was used to gain enough exposure to the heart while keeping rib integrity, which helped to avoid the exteriorization of the heart.

It is well known that successful development of HF post-MI in rat largely depends on the infarct size, which is related to the ligation site of the LCA. As early as 1979, Marc A. Pfeefer and his colleagues reported that LCA occlusion in rats could readily provide left ventricular free wall infarctions of varying sizes $^{18}$. To reduce the variation of infarct sizes and to develop a more stable HF model post-MI, LAD ligation has been commonly used in rats to induce left ventricular infarction for research purposes ${ }^{19}$. The major advantage of LAD occlusion is to allow for accurate ligation of this artery to induce a stable MI that can cause HF while keeping a much higher survival rate. In our procedure, we chose the ligation position 2.0-2.5 $\mathrm{mm}$ below the midpoint of the connection between the conus arteriosus and left atrial appendage, which proved to be successful and stable with less variations in LVEF among all the rats in the MI group. In addition, we individually determined the infarct size by TTC staining and the degree of fibrosis by Masson's staining, both of which have been widely used to evaluate this kind of model $20,21,22$. Meanwhile, the less variations of this model were also illustrated through morphological alterations and histopathology analyses.

We also studied some parameters related to $\mathrm{MI}$ and $\mathrm{HF}$ after 4 weeks of the LAD ligation to help confirm the development of HF after MI. Echocardiography has the ability to accurately and noninvasively measure ventricular function and assess causes of structural heart disease ${ }^{23}$. The LVEFs of the mice in the $\mathrm{Ml}$ group were all under $50 \%$ which could be considered as $\mathrm{HF}^{12}$. In line with these results, the concentration of NT-proBNP was increased accordingly which was also an important indicator of impairment of heart function. Ample evidence exists for inflammation and apoptosis in the ischemic heart 24,25 . Inflammation and heart failure are strongly interconnected and mutually reinforce each other ${ }^{26}$. In our study, we evaluated the significant increase in inflammation factors, IL-6 and TNF-a. The reninangiotensin-aldosterone system (RAAS) activated by renal hypoperfusion and sympathetic activation is a central feature in the pathophysiology of heart failure ${ }^{27}$. We evaluated the parameters involved in the RAAS and we found that the serum level of renin was significantly higher in the Ml group compared to the sham group, which further verified the development of HF.

There are two main points of emphasis during the procedure of LAD ligation. First, during the whole procedure and until they recover from anesthesia, provide a continuous supply of oxygen to the animals while also keeping them warm. Secondly, the position of ligation on the LAD has a key role in the infarct size and the degree of HF. The site 2.0-2.5 $\mathrm{mm}$ below the midpoint of the connection between the conus arteriosus and left atrial appendage is appropriate for a stable 
and successful HF model with less mortality rate. However, the limitation of the new HF model in rats is that the surgical procedure for developing MI without ventilation is technically demanding and challenging. The key step for achieving this model is to gain enough exposure of the heart to fully visualize the heart inside the thorax and limit the time the chest is open to 5 minutes, which requires more practice before the formal experiment.

\section{Disclosures}

The authors have nothing to disclose.

\section{Acknowledgments}

This model was developed with the support of Shandong Co-Innovation Center of Classic TCM Formula, Shandong Provincial Education Department. We would like to thank Dr. Lianfeng Wu for performing the echocardiography in this study.

\section{References}

1. Savarese, G., Lund, L.H. Global Public Health Burden of Heart Failure. Cardiac Failure Review. 3 (1), 7-11 (2017).

2. Ziaeian, B., Fonarow, G.C. Epidemiology and aetiology of heart failure. Nature Reviews Cardiology. 13 (6), 368-378 (2016).

3. Meijers, W.C., de Boer, R.A. Common risk factors for heart failure and cancer. CardiovascularResearch. 115 (5), 844-853 (2019).

4. Triposkiadis, F., Xanthopoulos, A., Butler, J. Cardiovascular Aging and Heart Failure: JACC Review Topic of the Week. Journal of the American College of Cardiology. 74 (6), 804-813 (2019).
5. Tanai. E., Frantz, S. Pathophysiology of Heart Failure. Comprehensive Physiology. 6 (1), 187-214 (2015).

6. Cahill, T.J., Kharbanda, R.K. Heart failure after myocardial infarction in the era of primary percutaneous coronary intervention: Mechanisms, incidence and identification of patients at risk. World Journal Of Cardiology. 9 (5), 407-415 (2017).

7. Houser, S.R. et al. Animal models of heart failure: a scientific statement from the American Heart Association. Circulation Research. 111 (1), 131-150 (2012).

8. Patten, R.D., Hall-Porter, M.R. Small animal models of heart failure: development of novel therapies, past and present. Circulation Heart Failure. 2 (2), 138-144 (2009).

9. Chen, J. et al. Ischemic Model of Heart Failure in Rats and Mice. Methods in Molecular Biology (Clifton, NJ). 1816, 175-182 (2018).

10. Chen, J., Ceholski, D.K., Liang, L., Fish, K., Hajjar, R.J. Variability in coronary artery anatomy affects consistency of cardiac damage after myocardial infarction in mice. American Journal of Physiology Heart and Circulatory Physiology. 313 (2), H275-h282 (2017).

11. Goldman, S., Raya, T.E. Rat infarct model of myocardial infarction and heart failure. Journal of Cardiac Failure. 1 (2), 169-177 (1995).

12. Ponikowski, P. et al. 2016 ESC Guidelines for the diagnosis and treatment of acute and chronic heart failure: The Task Force for the diagnosis and treatment of acute and chronic heart failure of the European Society of Cardiology (ESC). Developed with the special contribution of the Heart Failure Association (HFA) of the 
ESC. European Journal of Heart Failure. 18 (8), 891-975 (2016).

13. Gao, E. et al. A novel and efficient model of coronary artery ligation and myocardial infarction in the mouse. Circulation Research. 107 (12), 1445-1453 (2010).

14. van den Bos, E.J., Mees, B.M., de Waard, M.C., de Crom, R., Duncker, D.J. A novel model of cryoinjury-induced myocardial infarction in the mouse: a comparison with coronary artery ligation. American Journal of Physiology Heart and Circulatory Physiology. 289 (3), H1291-1300 (2005).

15. Lindsey, M.L. et al. Guidelines for experimental models of myocardial ischemia and infarction. American Journal of Physiology Heart and Circulatory Physiology. 314 (4), H812-h838 (2018).

16. Henri, O. et al. Selective Stimulation of Cardiac Lymphangiogenesis Reduces Myocardial Edema and Fibrosis Leading to Improved Cardiac Function Following Myocardial Infarction. Circulation. 133 (15), 1484-1497; discussion 1497 (2016).

17. Fu, Y.H. et al. A novel rat model of chronic heart failure following myocardial infarction. Methods and Findings in Experimental and Clinical Pharmacology. 31 (6), 367-373 (2009).

18. Pfeffer, M.A. et al. Myocardial infarct size and ventricular function in rats. Circulation Research. 44 (4), 503-512 (1979).

19. Samsamshariat, S.A., Samsamshariat, Z.A., Movahed, M.R. A novel method for safe and accurate left anterior descending coronary artery ligation for research in rats. Cardiovascular Revascularization Medicine: Including Molecular Interventions. 6 (3), 121-123 (2005).
20. Redfors, B., Shao, Y., Omerovic, E. Myocardial infarct size and area at risk assessment in mice. Experimental and Clinical Cardiology. 17 (4), 268-272 (2012).

21. Rüder, C. et al. Combinatorial G-CSF/AMD3100 treatment in cardiac repair after myocardial infarction. PloSOne. 9 (8), e104644 (2014).

22. Valentin, J., Frobert, A., Ajalbert, G., Cook, S., Giraud, M.N. Histological Quantification of Chronic Myocardial Infarct in Rats. Journal of Visualized Experiments. (118) (2016).

23. Kirkpatrick, J.N., Vannan, M.A., Narula, J., Lang, R.M. Echocardiography in heart failure: applications, utility, and new horizons. Journal of the American College of Cardiology. 50 (5), 381-396 (2007).

24. Teringova, E., Tousek, P. Apoptosis in ischemic heart disease. Journal of Translational Medicine. 15 (1), 87 (2017).

25. Wang, X., Guo, Z., Ding, Z., Mehta, J.L. Inflammation, Autophagy, and Apoptosis After Myocardial Infarction. Journal of the American Heart Association. 7 (9) (2018).

26. Van Linthout, S., Tschöpe, C. Inflammation - Cause or Consequence of Heart Failure or Both? Current Heart Failure Reports. 14 (4), 251-265 (2017).

27. Orsborne. C., Chaggar, P.S., Shaw, S.M,. Williams, S.G. The renin-angiotensin-aldosterone system in heart failure for the non-specialist: the past, the present and the future. Postgraduate Medical Journal. 93 (1095), 29-37 (2017). 